\title{
Capture the time when plants reach their maximum body size by using the beta sigmoid growth equation
}

\author{
Pei-Jian Shi ${ }^{a, *}$, Lei Chen ${ }^{\mathrm{b}}$, Cang Hui ${ }^{\mathrm{c}, \mathrm{d}}$, Henri D. Grissino-Mayer ${ }^{\mathrm{e}}$ \\ a Collaborative Innovation Center of Sustainable Forestry in Southern China of Jiangsu Province, Bamboo Research Institute, Nanjing Forestry University, \\ 159 Longpan Road, Xuanwu District, Nanjing 210037, China \\ ${ }^{\mathrm{b}}$ Graduate School of Environmental Science, Hokkaido University, N19W8, Sapporo 060-0819, Japan \\ c Centre for Invasion Biology, Department of Mathematical Sciences, Stellenbosch University, Matieland, South Africa \\ ${ }^{\mathrm{d}}$ Mathematical and Physical Biosciences, African Institute for Mathematical Sciences, Cape Town, South Africa \\ e Department of Geography, The University of Tennessee, Knoxville, TN 37996, USA
}

\section{A R T I C L E I N F O}

\section{Article history:}

Received 29 August 2015

Received in revised form 9 September 2015

Accepted 10 September 2015

Available online 11 November 2015

\section{Keywords:}

Beta sigmoid function

Data fitting

Growth equation

Growth rate

\begin{abstract}
A B S T R A C T
Of the many mathematical models proposed for capturing the dynamics of plant growth, the beta sigmoid function (BSF) is the newest and consequently is not well known to ecologists. A recent software package based on the Microsoft Excel macro, LEAF-E, was designed to promote the use of BSF, even though the performance of BSF and other growth models had not been compared. We developed R functions for fitting the BSF with a freer option for choosing the parametric number, and illustrated their performance using simulated data generated by four equations (the exponential, logistic, Gompertz, and von Bertalanffy equations), as well as dry weights of six crop species measured in growing seasons. Compared to other growth models, the BSF allowed for both symmetric and asymmetric growth curves, and thus the simulated data modeled the actual data quite well. It was demonstrated that the BSF was better than the above four traditional growth equations. In addition, the $\mathrm{R}$ functions developed here can facilitate future data fitting and model comparison for capturing plant growth dynamics. And the time when plants reach their maximum body size can be accurately obtained by using the BSF.
\end{abstract}

() 2015 Elsevier B.V. All rights reserved.

\section{Introduction}

Plant growth models are at the heart of plant metrics (Paine et al., 2012), where the dynamics of weight or height of focal plant species can be described (Shi et al., 2013). The growth of some plant tissues and organs can also be depicted by such models, such as the mean number of xylem cells, leaf length, and fruit size during the growing seasons (Huang et al., 2011; Voorend et al., 2014). Recently, a nonlinear regression-based tool, LEAF-E (Voorend et al., 2014), a macro within the Microsoft Excel platform, was devised for estimating the parameters of the beta sigmoid function (BSF), a model proposed by Auzanneau et al. (2011) for describing leaf growth, originally proposed by Yin et al. (2003). Here, we aimed to: (1) provide R functions for data fitting (R Core Team, 2015); (2) evaluate the performance of BSF using simulated data from four traditional growth equations (exponential, logistic, Gompertz and von Bertalanffy equations); and (3) evaluate the performance of

\footnotetext{
* Corresponding author. Tel.: +86 25 85427231; fax: +86 2585427487.

E-mail address: peijianshi@gmail.com (P.-J. Shi).
}

BSF using actual plant growth data. In addition, we found that the BSF used by Voorend et al. (2014) could not be directly derived from that of Yin et al. (2003) as they had stated. Our equation was directly derived from the latter, and performed well both for simulated data and actual growth data of plants.

\section{Materials and methods}

\subsection{Simulated and real datasets}

We used the following growth equation to simulate plant growth data:

(i) Exponential equation

$$
w= \begin{cases}w_{0} \exp (r t) & \text { if } t<t_{e} \\ w_{\max } & \text { if } t \geq t_{e}\end{cases}
$$

where $w$ represents the weight at time $t ; w_{0}$ is the initial weight at $t=0 ; w_{\max }$ is the maximal weight at time $t=t_{e}$; and $r$ is the instantaneous growth rate. 

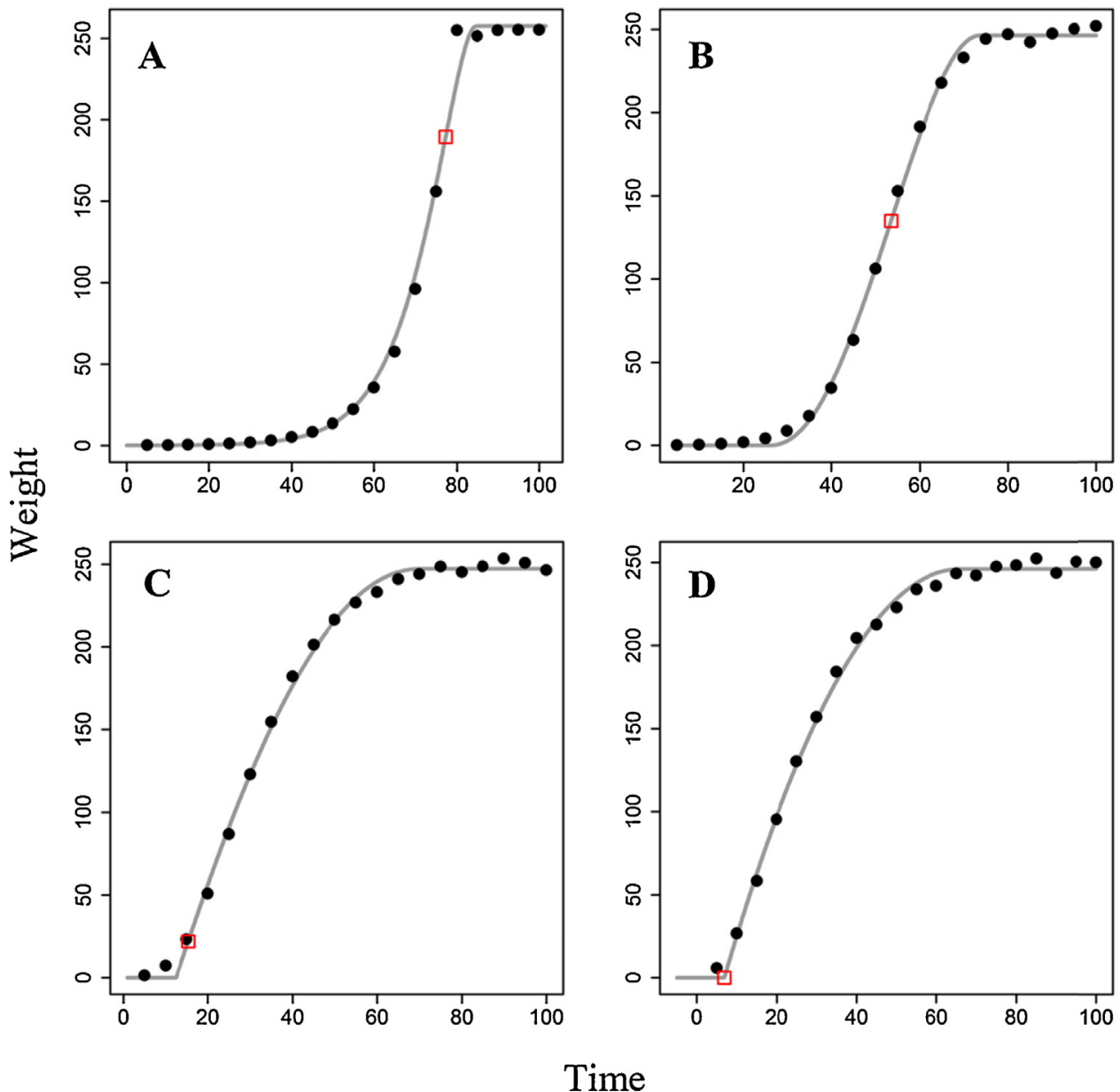

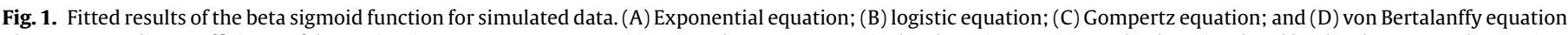

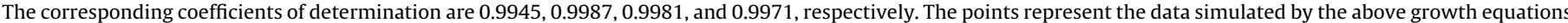
with $C V=1 \%$ noise, and the gray curves are predicted values from the beta sigmoid function. Here, CV denotes the coefficient of variation.

(ii) Logistical equation

$$
w=\frac{w_{\max }}{1+\left(\left(w_{\max } / w_{0}\right)-1\right) \exp (-r t)}
$$

(iii) Gompertz equation

$$
w=w_{\max } \exp \left[-\ln \left(\frac{w_{\max }}{w_{0}}\right) \cdot \exp (-r t)\right]
$$

(iv) von Bertalanffy equation

$$
w=w_{\max }\left\{1-\left[1-\left(\frac{w_{0}}{w_{\max }}\right)^{1 / 4}\right] \cdot \exp \left[\frac{-a t}{4 w_{\max }^{1 / 4}}\right]\right\}^{4}
$$

Here, $a$ is a parameter. It is a special case of the generalized von Bertalanffy equation which is commonly referred to as the “ontogenetic growth model" (Shi et al., 2013).
We set $w_{0}=0.1$, and $w_{\max }=250$ for all four equations during the simulations. For the exponential equation, $r=0.098, t_{e}=80$; for the logistic equation, $r=0.15$; for the Gompertz equation, $r=0.08$; for the von Bertalanffy equation, $a=1$.1. These parameters were set just to make comparison apparent when $0<t<250$. In fact, we could change these parameters to other values and they will not affect the main results when using the BSF to fit the simulated data. The time was set to range from 5 to 250 with an increment of 5 . To validate the model for data with noise, we added a normal random number with zero mean and $1 \%$ standard error to the simulated weights from the above models at each sampling point.

We chose six species of crops from the study by Shi et al. (2013). These crops were planted in field on 27 June, 2011. Measurements of total plant fresh and dry weights were performed on 15 dates

\begin{tabular}{|c|c|c|c|c|c|c|c|}
\hline Common name & Latin name & $c_{m}$ & $t_{m}$ & $t_{e}$ & RSS & $\chi^{2}$ & $R^{2}$ \\
\hline Kidney bean & Phaseolus vulgaris L. & 0.34719 & 47.44 & 71.58 & 2.69 & 0.36 & 0.9910 \\
\hline Adzuki bean & Vigna angularis (Willd.) Ohwi et Ohashi & 0.899 & 60.98 & 73.16 & 2.47 & 4.29 & 0.9981 \\
\hline Mungbean & Vigna radiata (L.) R. Wilczek & 1.06537 & 67.64 & 84.31 & 11.26 & 0.92 & 0.9966 \\
\hline Cotton & Gossypium spp. & 3.16052 & 71.84 & 85.22 & 120.53 & 17.90 & 0.9938 \\
\hline Sweet sorghum & Sorghum bicolor (L.) Moench & 6.49894 & 66.33 & 80.09 & 516.39 & 10.17 & 0.9947 \\
\hline Corn & Zea mays $\mathrm{L}$. & 9.25776 & 70.73 & 89.42 & 1360.29 & 14.69 & 0.9946 \\
\hline
\end{tabular}

Table 1

Parametric estimates and the goodness-of-fit by using the beta sigmoid function to fit the dry weight of six crop species.

Here, $t_{b}=0$. 
through the growing season. For each species, 20 samples were randomly chosen during each investigation. The total dry weight of plants indicates the weight sum of the above- and under-ground parts. Here, we used the mean dry weight data for examining the BSF.

\subsection{Beta sigmoid function (BSF)}

The beta distribution function is usually used for capturing different distribution types. It was introduced to describe the effect of temperature $(T)$ on the developmental rate $(r)$ of crops by Yin et al. (1995):

$r=c_{m}\left[\left(\frac{T_{c}-T}{T_{c}-T_{m}}\right)\left(\frac{T-T_{b}}{T_{m}-T_{b}}\right)^{\left(T_{m}-T_{b}\right) /\left(T_{c}-T_{m}\right)}\right]^{\delta}$

where $T_{b}$ represents the base temperature at which the development rate equals $0 ; T_{c}$ is the ceiling temperature at which the developmental rate also equals $0 ; T_{m}$ is a temperature at which the developmental rate can reach its maximum value $\left(=c_{m}\right)$; and $\delta$ is a scaling constant. It is necessary to point out that Eq. (5) should be applicable to both the developmental rate and growth rate of
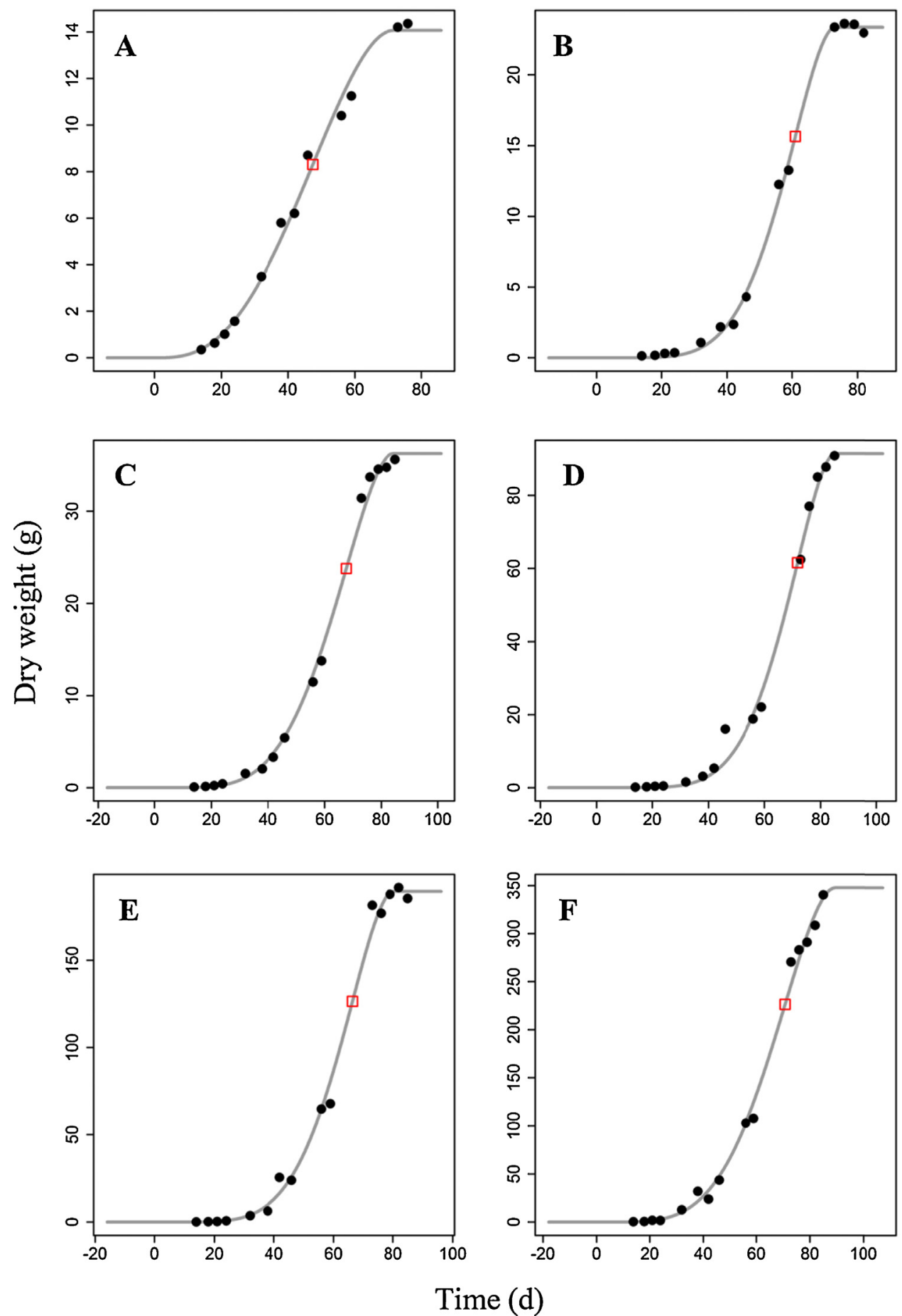

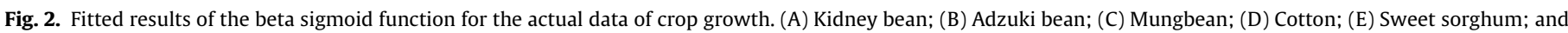
(F) Corn. The points represent the observations of dry weights; and the gray curves represent the predicted valued by using the beta sigmoid function. 
crops. Similar temperature-dependent developmental rate models also have been considered to apply to both the developmental rate and growth rate of poikilotherms and bacteria (e.g., Logan et al., 1976; Sharpe and DeMichele, 1977; Ratkowsky et al., 2005). Yin et al. (2003) obtained a differential function based on the above beta distribution function:

$\frac{d w}{d t}=c_{m}\left[\left(\frac{t_{e}-t}{t_{e}-t_{m}}\right)\left(\frac{t-t_{b}}{t_{m}-t_{b}}\right)^{\left(t_{m}-t_{b}\right) /\left(t_{e}-t_{m}\right)}\right]^{\delta}$

First, the left side of Eq. (5) was replaced by a derivative, $d w / d t$, where $w$ represents weight of plants and $t$ represents time. Second,
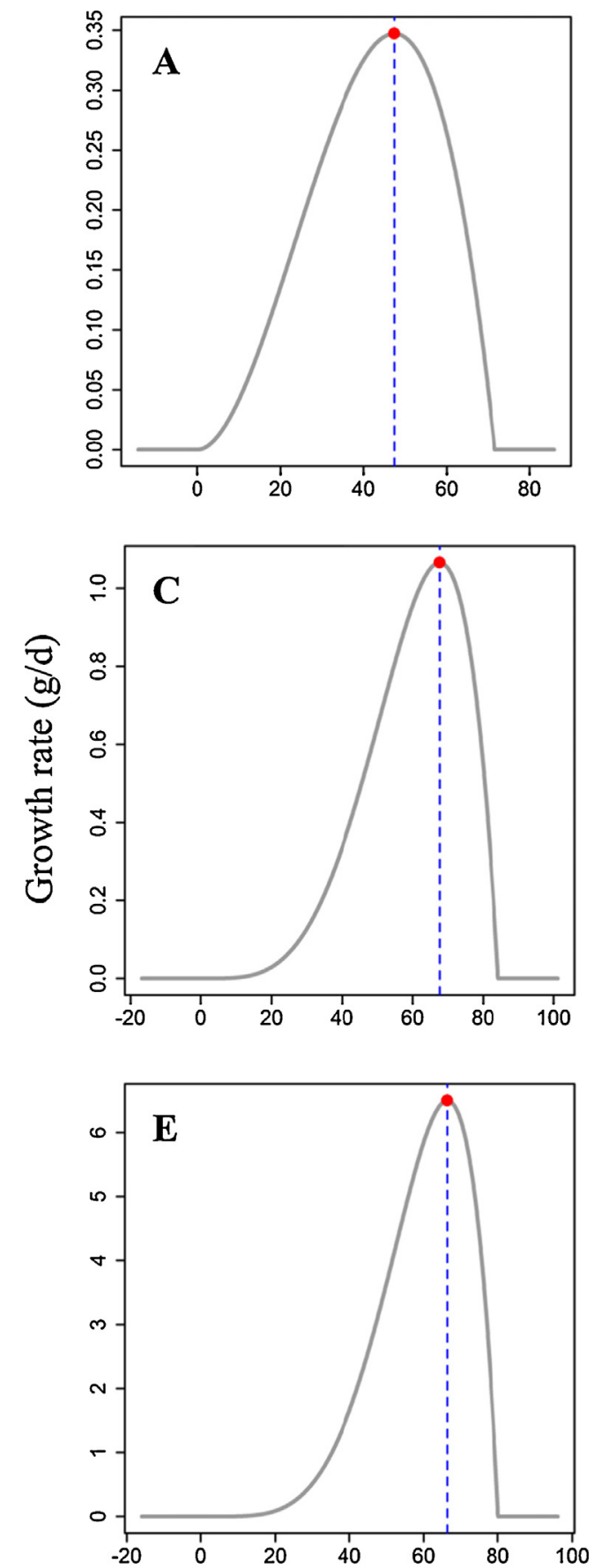

on the right side of Eq. (5), temperature $T$ was replaced by time $t$. Third, $t_{b}$ and $t_{e}$ represent the beginning and end of the growth period at which growth rates both equal 0 . Finally, $c_{m}$ represents the maximal growth rate at the time $t_{m}$ and $\delta$ is still a constant When $\delta$ differs from one and zero, Eq. (6) does not have an analytical solution. To simplify it, let $\delta=1$ and $t_{b}=0$ and we have a simplified BSF (Yin et al., 2003):

$w=c_{m} \cdot t \cdot\left(\frac{2 t_{e}-t_{m}-t}{2 t_{e}-t_{m}}\right)\left(\frac{t}{t_{m}}\right)^{t_{m} /\left(t_{e}-t_{m}\right)}$
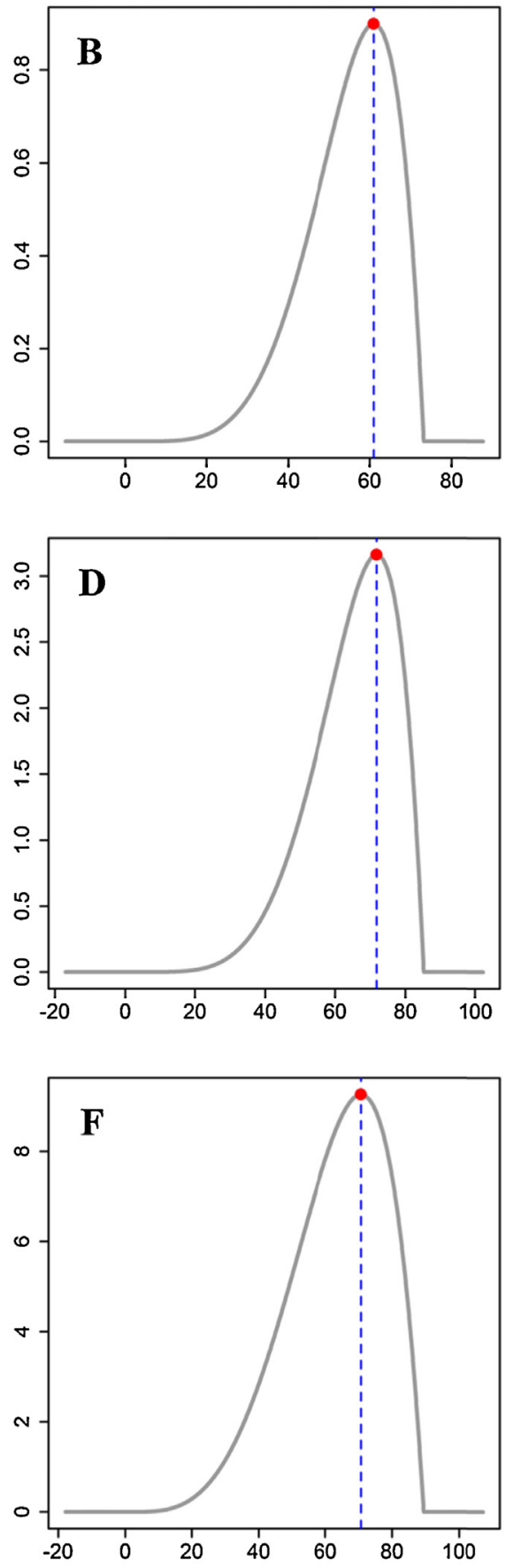

Time (d)

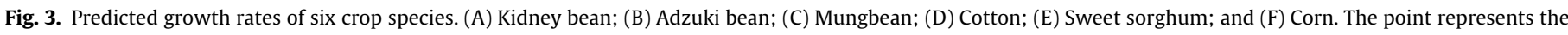
maximal growth rate. 
Note that when $t=0, w=0$ in the equation. This growth equation can describe many real datasets of plant growth, but it lacks fitting flexibility. In addition, in most cases, when $t=0, w$ does not equal 0 . It is also necessary to point out that Eq. (7) omitted a constant because it came from the integral of Eq. (6). To render the above beta sigmoid function to be more flexible, we only assumed $\delta=1$ in Eq. (6). After integrating Eq. (6), we obtain:

$w=c_{m} \cdot\left(t-t_{b}\right) \cdot \frac{2 t_{e}-t_{m}-t}{2 t_{e}-t_{m}-t_{b}}\left(\frac{t-t_{b}}{t_{m}-t_{b}}\right)^{\left(t_{m}-t_{b}\right) /\left(t_{e}-t_{m}\right)}$

Here, we also omitted a constant on the right side of the above equation because we assumed $w=0$ on the condition of $t=t_{b}$. Eq. (8) is slightly different from Eq. (1) published in Voorend et al. (2014). However, we found that these two equations could produce a similar growth curve. Note: there was a misprint in Eq. (11) of Yin et al. (2003), but our Eq. (8) was directly derived from their Eq. (7) and had no relationship with their Eq. (11). Here, we used Eq. (8) and developed a list of $\mathrm{R}$ functions (see the Online Supplementary data 1 and 2) to fit the simulated and real datasets.

\section{Results and discussion}

Fig. 1 showed the results of the fitted weights from the BSF and the simulated data (see Methods for details). Although the BSF can capture the dynamics well, we observed slight differences of weight between the fitted BSF and simulated data, especially at the initial and final growth stages. As little attention has been paid to bridging the BSF to these existing growth equations, it is difficult to judge whether the BSF could have under- or over-estimated the actual weight at the two growth stages. A test using real data is needed. Fig. 2 showed the fitted BSF with the real dry weights of six crop species in growing reasons, with their grow rates predicted (Fig. 3; Table 1).

Theoretically, the logistic model gives a symmetric growth rate curve (Yin et al., 2003; Paine et al., 2012). However, none showed a symmetric growth rate curve (Fig. 3). That is, the logistical model could lead to misleading conclusions on predicting the growth rate. In addition, the logistic model over-estimated the maximum dry weight of these crop species, even though it estimated a lower asymptotic dry weight than those from the Gompertz and von Bertalanffy equations (Shi et al., 2013). In all these cases, the BSF provided a more reliable estimation of the asymptotic dry weight. However, for reliable parameter estimates, the BSF has a high demand for data quality, especially for the dry weight at the end of the growing stage.

Overall, the logistical equation is better than the exponential, Gompertz, and von Bertalanffy equations (Shi et al., 2013), but it is worse than the BSF. The logistic equation provided a too large estimate on the maximum asymptotic value for body size of plants. The BSF predicted a reasonable maximum asymptotic value for body size, and also provided an accurate estimate on the time of reaching the maximum body size.

\section{Conclusions}

We proposed a BSF based on Yin et al. (2003), slightly different from that proposed by Voorend et al. (2014), although both can produce similar growth curves. We developed a list of $\mathrm{R}$ functions for fitting the parameters of BSF. We found that the BSF can capture the dynamics of plant growth following traditional growth equations, such as the exponential, logistic, Gompertz, and von Bertalanffy equations. The uniqueness of our BSF is that it permits an asymmetric growth curve and provides a better fit to the actual plant growth data. It provided the most reliable estimates of the asymptotes in the real observations. The $\mathrm{R}$ functions could further promote the wide use of the BSF in simulating plant growth.

\section{Competing interests}

The authors declare that they have no competing interests.

\section{Authors' contributions}

PJS and $\mathrm{CH}$ designed this study. PJS and LC developed R functions. PJS, $\mathrm{CH}$ and HDGM wrote this manuscript. The language was further smoothed by HDGM. All authors read and approved the final manuscript.

\section{Acknowledgements}

We are deeply thankful to the editor-in-chief, Brian D. Fath, and an anonymous reviewer for their valuable comments. PJS was partially supported by the National Natural Science Foundation of China (grant number 31400348), the PAPD of Jiangsu Province; $\mathrm{CH}$ was supported by the National Research Foundations of South Africa (grant number 76912 and 81825).

\section{Appendix A. Supplementary data}

Supplementary data associated with this article can be found, in the online version, at http://dx.doi.org/10.1016/j.ecolmodel.2015. 09.012.

\section{References}

Auzanneau, J., Huyghe, C., Escobar-Gutiérrez, J., Julier, B., Gastal, F., Barre, P., 2011. Association study between the gibberellic acid insensitive gen and leaf length in a Lolium perenne L. synthetic variety. BMC Plant Biol. 11, 183.

Huang, J.G., Bergeron, Y., Zhai, L.H., Denneler, B., 2011. Variation in intra-annual radial growth (xylem formation) of Picea mariana (Pinaceae) along a latitudinal gradient in western Quebec, Canada. Am. J. Bot. 98, 792-800.

Logan, J.A., Wollkind, D.J., Hoyt, S.C., Tanigoshi, L.K., 1976. An analytic model for description of temperature dependent rate phenomena in arthropods. Environ. Entomol. 5, 1133-1140.

Paine, C.E.T., Marthews, T.R., Vogt, D.R., Purves, D., Rees, M., Hector, A., Turnbull, L.A., 2012. How to fit nonlinear plant growth models and calculate growth rates: an update for ecologists. Methods Ecol. Evol. 3, 245-256.

R Core Team, 2015. R: A Language and Environment for Statistical Computing. R Foundation for Statistical Computing, Vienna, Austria http://www.R-project. org/.

Ratkowsky, D.A., Olley, J., Ross, T., 2005. Unifying temperature effects on the growth rate of bacteria and the stability of globular proteins. J. Theor. Biol. 233, 351-362.

Sharpe, P.J.H., DeMichele, D.W., 1977. Reaction kinetics of poikilotherm development. J. Theor. Biol. 64, 649-670.

Shi, P.J., Men, X.Y., Sandhu, H.S., Chakraborty, A., Li, B.L., Ouyang, F., Sun, Y.C., Ge, F., 2013. The "general" ontogenetic growth model is inapplicable to crop growth. Ecol. Model. 266, 1-9.

Voorend, W., Lootens, P., Nelissen, H., Roldán-Ruiz, I., Inzé, D., Muylle, H., 2014. LEAFE: a tool to analyze grass leaf growth using function fitting. Plant Methods 10, 37.

Yin, X., Goudriaan, J., Lantinga, E.A., Vos, J., Spiertz, H.J., 2003. A flexible sigmoid function of determinate growth. Ann. Bot. 91, 361-371.

Yin, X., Kropff, M.J., McLaren, G., Visperas, R.M., 1995. A nonlinear model for crop development as a function of temperature. Agric. For. Meteorol. 77, 1-16. 